\title{
An efficient tomographic inversion approach for urban mapping using meter resolution SAR image stacks
}

\author{
Yuanyuan Wang ${ }^{(1)}$, Xiao Xiang Zhu ${ }^{(1,2)}$, Richard Bamler ${ }^{(1,2)}$ \\ (1) Lehrstuhl für Methodik der Fernerkundung, Technische Universität München, Arcisstraße 21, 80333 \\ Munich, Germany \{Email: wang@bv.tum.de\} \\ (2) Remote Sensing Technology Institute (IMF), German Aerospace Center (DLR), Oberpfaffenhofen, \\ 82234 Weßling, Germany \{Email: xiao.zhu@dlr.de
}

\begin{abstract}
This article describes an efficient approach of multi-dimensional synthetic aperture radar (SAR) imaging for urban mapping. The proposed approach is an integration of tomographic SAR inversion and the well-known persistent scatterer interferometry (PSI). It consists of three steps: first, a global estimation of the topography and motion parameters using efficient algorithms like PSI; second, a single and double scatterer discrimination step based on the results of the first step; and lastly, a tomographic SAR inversion, performed on the pre-classified double scatterers, using the prior knowledge obtained in the first step, retrieving the topography and motion parameters of both scatterers.

The proposed approach has been tested on a dozen of TerraSAR-X high resolution spotlight image stacks. In this paper, examples from Las Vegas and Berlin are presented. The results are comparable to the one obtained by the most computationally expensive tomographic SAR algorithms (e.g. SL1MMER) only, and saves computational time by a factor of fifty.
\end{abstract}


Keywords: SAR tomography, urban mapping, TerraSAR-X, synthetic aperture radar, SL1MMER 


\section{INTRODUCTION}

\section{A. TomoSAR and PSI}

Tomographic synthetic aperture radar (SAR) inversion includes SAR tomography (TomoSAR) [1] and differential SAR tomography (D-TomoSAR) [2] [3] [4]. Because of their layover resolving capability, they are often used for urban 3D topographic reconstruction, forest structure imaging, and ground or building deformation monitoring. As an extension of TomoSAR, D-TomoSAR introduces an additional motion term in the TomoSAR system model, allowing the retrieval of the motion parameters of each scatterer in a resolution cell. The D-TomoSAR system model can be expressed as follows:

$$
g_{n}=\int_{\Delta s} \gamma(s) \exp \left(-j 2 \pi\left(\xi_{n} s+\frac{2 d\left(s, t_{n}\right)}{\lambda}\right)\right) d s
$$

where $g_{n}$ is the complex pixel value in the $n$th single look complex (SLC) SAR image, $s$ represents the elevation direction, $d\left(s, t_{n}\right)$ is the displacement as a function of the elevation and the acquisition time $t_{n}, \lambda$ is the wavelength of SAR system, $\xi_{n}=-2 b_{n} /(\lambda r)$ which is the so called "spatial frequency", and is proportional to the baseline $b_{n}$ and range $r$, and $\gamma(s)$ is the complex reflectivity profile along the elevation direction of that pixel. Solving the reflectivity $\gamma(s)$ is essentially a spectral estimation problem. In case of solving it parametrically, the solution is in addition subject to a multi-dimensional search in the elevation and motion parameters solution space.

Persistent scatterer interferometry (PSI) [5] assumes maximum a single dominant scatterer in a pixel. Under such assumption, $\gamma(s)$ in Equation (1) is modeled as a delta function with constant amplitude. Assuming linear and seasonal motion models (common in urban area), the PSI system model can be simplified as: 


$$
g_{n}=A \exp \left(-j 2 \pi\left(\xi_{n} s+\eta_{n} v+\omega_{n} a\right)\right)
$$

where $A$ is the amplitude of the point scatterer which is usually dropped in the estimation, $\eta_{n}=2 t_{n} / \lambda$ is the temporal frequency modeling linear movement and $\omega_{n}=2 \sin \left(2 \pi\left(t_{n}-t_{0}\right)\right) / \lambda$ is the temporal frequency modeling seasonal motion. $v, a$ are the velocity of linear motion and the amplitude of periodic motion, respectively. Solving the unknown elevation and the motion parameters is to match the phase on both sides of Equation (2), which can be efficiently solved by maximizing the ensemble coherence (periodogram) [6]. Because of the simplification in the system model, PSI is an efficient method for deformation monitoring of large areas.

\section{B. Integration of TomoSAR and PSI}

As the resolution of SAR images going increasingly higher, it on one hand provides us enormous details, and on the other hand increases the computational burden. This is especially true for monitoring an entire urban area using D-TomoSAR. Because it is a spectral estimation problem whose computational efficiency is mainly restricted by:

- Dimension of the spectral estimation problem for each pixel. Usually 3D is applied (elevation, linear motion and periodic motion) [7];

- Extent of the parameter solution range, namely the search range in each dimension of the solution space;

- The spectral estimation algorithms being applied, such as periodogram (economic), SVD-Wiener [4] (little bit more expensive) and SL1MMER [8] (much more expensive).

To reduce the computation, we propose to integrate PSI into the D-TomoSAR inversion, with the following considerations:

- PSI needs provide a reasonable atmospheric phase screen (APS) estimation

- PSI should provide the prior knowledge about the dimension of the problem (i.e. the motion model), and also the search range in each dimension of the solution space; 
- more expensive methods such as SVD-Wiener and SL1MMER shall only be applied to the pixels that probably contain more than one scatterer;

- Algorithms with super-resolving capability such as SL1MMER shall only be applied when super-resolution is required.

\section{Summary}

The proposed TomoSAR inversion approach is an integration of PSI and D-TomoSAR. The processing flowchart is shown in Figure 1. It follows three steps: first order spectral estimation, single and double scatterer(s) discrimination, and higher order spectral estimation. A near-operational processor is implemented by combining the PSI, SVD-Wiener, and SL1MMER developed in the Remote Sensing Technology Institute of the German Aerospace Center (DLR).

The rest of the article will describe each step in this approach. The details of PSI, SVD-Wiener, and SL1MMER will not be addressed, and can be found in [4] [5] and [8]. Focus will be put on the integration of PSI and D-TomoSAR, especially the discrimination of pixels with single or double scatterer(s). Results using TerraSAR-X high resolution spotlight data are present in Chapter III. 


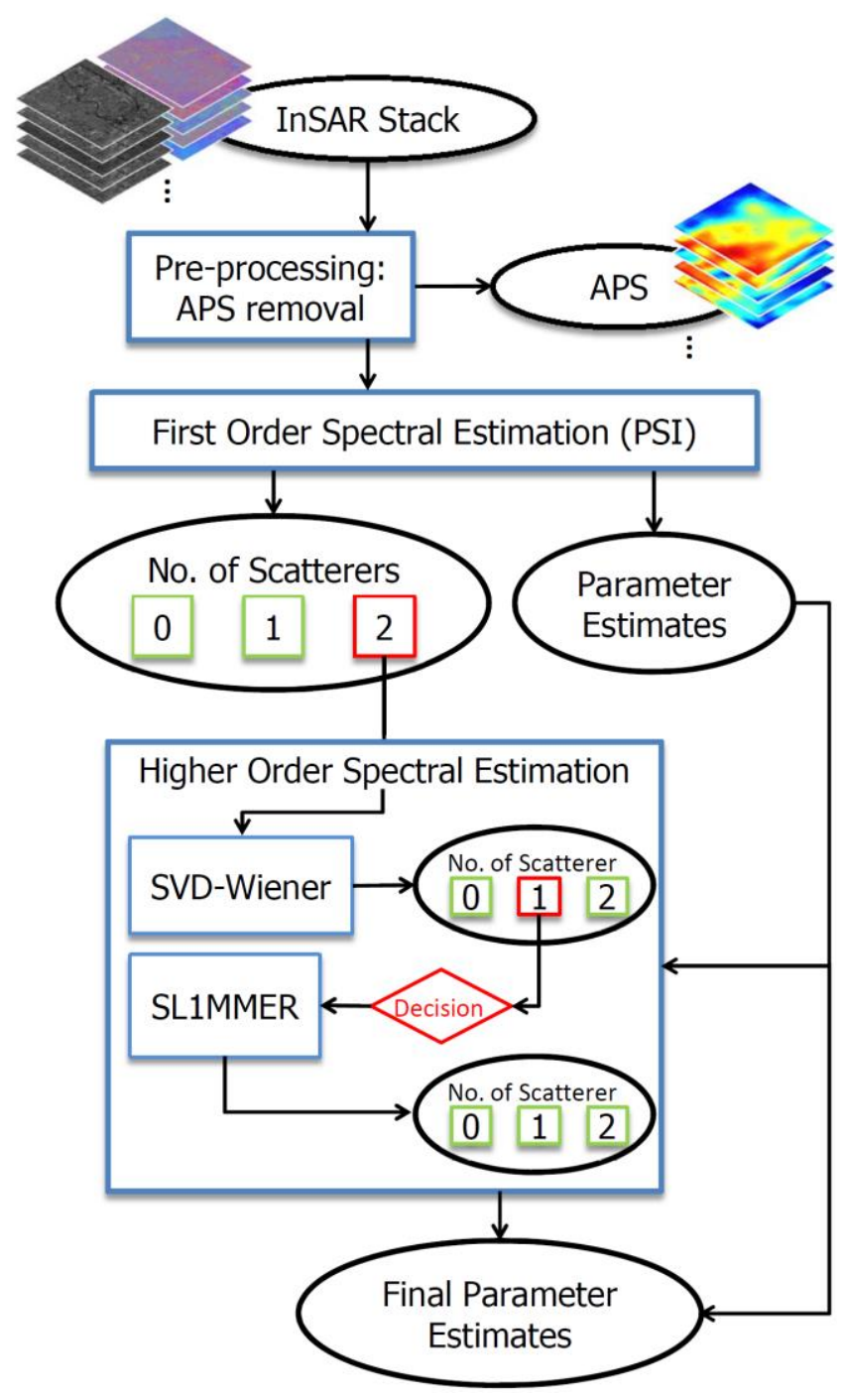

Figure 1. Processing flow chart. Black ellipses represent input/output data. Blue rectangles are processes/algorithms. The red diamond represents decision. The green rectangles in the black ellipses are accepted results, while those red ones are results that need to be passed to the next step.

\section{TOMOSAR PROCESSING CHAIN}

The proposed approach is summarized in Figure 1. Firstly, APS is removed from the co-registered image stack in the PSI-type pre-processing; then, first order spectral estimation (i.e. single scatterer model) is applied on all the pixels, obtaining the estimates of the model parameters. Based on these estimates, amplitude dispersion index and pixel brightness, a pre-discrimination of the number of scatterers in each pixel is then obtained. As we limit the maximum number of scatterers to two, all the 
pixels shall fall into one of the three classes: no, single, or double scatterer(s). The pre-discrimination follows two steps: the Bayesian decision rule is firstly applied to discard pixels with no scatterer, and followed by a support vector machine (SVM) to separate pixels with single scatterer and double scatterers. The pixels detected with double scatterers (upper red box in Figure 1) are passed to higher order spectral estimation. They are firstly processed with SVD-Wiener. Some of them are confirmed as pixels containing double scatterers and the rest are rejected as having either single or no scatterer. Because SVD-Wiener has almost no super-resolution capability, among those pixels rejected as single scatterers (lower red box in Figure 1), some actually contain double scatterers. Therefore, after a decision (red diamond in Figure 1) on the elevation distance, some of them are passed to the SL1MMER algorithm with super-resolution capability. The following text in this Chapter will explain each step in detail.

\section{A. Pre-processing}

The purpose of pre-processing is to estimate and further remove the APS from a co-registered complex SAR image stack. The image stacking is done by the PSI-GENESIS system [9] of DLR. The APS is removed using a similar approach as that in the standard PSI processing and also the "spatial difference" (SD) method mentioned in [10]. Since the APS is spatially smooth varying, the pre-processing is performed on the downsampled image stacks to reduce computational cost. Note that the pixel selection, and parameter estimates in the pre-processing do not affect/replace any procedures in the following D-TomoSAR inversion steps. The basic pre-processing procedures are listed as follows.

1. Downsample images in azimuth and range direction

2. Build redundant network from spatially differential measurement in the downsampled image stacks

3. Estimate model parameters of the differential measurements in the network, assuming single scatterer model with linear and seasonal motion

4. Integration of differential topography estimates, and remove the corresponding phase contribution from the original interferometric phase 
5. Unwrap the remaining residual phase in azimuth and range direction

6. Apply temporal high pass filter and spatial low pass filter to the unwrapped phase stack. This results the APS in the downsampled image size

7. Upsample the APS to the original image size, and remove from the original phase

\section{B. First order spectral estimation}

The first order spectral estimation refers to a special case of the D-TomoSAR problem considering only a single scatterer in each range-azimuth pixel. In the proposed processing chain, it is solved by maximizing the periodogram [11] of the amplitude-normalized measurements [5]:

$$
\{\hat{s}, \hat{v}, \hat{a}\}=\arg \max \left\{\left|\frac{1}{N} \sum_{n=1}^{N} \exp \left(-j 2 \pi\left(\xi_{n} s+\eta_{n} v+\omega_{n} a\right)\right) \times \frac{g_{n}^{*}}{\left|g_{n}\right|}\right|\right\}
$$

where $N$ is the total number of interferograms, * stands for the complex conjugate operator.

After applying the estimation on every pixel, we obtain the estimates of the elevation and motion parameters related to a reference point that we assume stable. Median and maximum filters are applied on the range-azimuth image of the estimates to obtain the prior for higher order spectral estimation. In addition, the following quantities are also recorded:

- Pixel brightness

- Amplitude dispersion index (ADI).

- Likelihoods of the single scatterer model and no-scatterer model w.r.t. the measurements $g_{n}$.

Under a circular Gaussian assumption of the measurements with covariance $\varepsilon$, the log-likelihood of single scatterer model $\left(H_{1}\right)$ is simply the sum of squared residuals [4]:

$$
\ln p\left(\mathbf{g} \mid H_{1}\right)=\ln \left(\frac{1}{\pi^{N}|\varepsilon|^{2 N}}\right)-\frac{1}{|\varepsilon|^{2}} \sum_{i=1}^{N}\left|g_{n}-\hat{A} \exp \left(-j 2 \pi\left(\xi_{n} \hat{s}+\eta_{n} \hat{v}+\omega_{n} \hat{a}\right)\right)\right|^{2}
$$


and for no-scatterer model $\left(H_{0}\right)$, it is the sum of squared measurements:

$$
\ln p\left(\mathbf{g} \mid H_{0}\right)=\ln \left(\frac{1}{\pi^{N}|\varepsilon|^{2 N}}\right)-\frac{1}{|\varepsilon|^{2}} \sum_{i=1}^{N}\left|g_{n}\right|^{2}
$$

\section{Number of scatterers discrimination}

Discriminating pixels with different numbers of scatterers is a classification problem. One of the optimum detection strategies is the likelihood ratio test, if the assumption of the underlying models is correct. However, such detection strategy requires the likelihood to the double scatterer model $\left(H_{2}\right)$, and hence is not feasible before the TomoSAR inversion. Therefore, in order to pre-discriminate the pixels before a TomoSAR inversion, our method is a two-step approach, comprising both theoretical and empirical parts.

Firstly, pixels without scatterer are discriminated following the Bayesian decision rule with penalized likelihood criterion:

$$
\frac{p\left(\mathbf{g} \mid H_{0}\right)}{p\left(\mathbf{g} \mid H_{1}\right) \exp (-A I C)} \frac{P_{0}}{P_{1,2}}>1
$$

The $H_{0}$ and $H_{1}$ likelihood of each pixel are compared, taking into account the Akaike information criterion (AIC) [4]. $P_{0}$ and $P_{1,2}$ are the prior probabilities of pixels with no scatterer and with single or double scatterer(s) respectively. Close to $100 \%$ detection rate and $0 \%$ false alarm rate can be achieved in this stage, because the $H_{1}$ likelihood of the pixels with double scatterers are much higher than their $H_{0}$ likelihood. This is proven in the real data experiment presented in Section IIIA.

The remaining pixels need to be further classified into single and double scatterer(s). Since the $H_{2}$ likelihood is not available at this stage, a few other easily accessible features associated with each pixel are used for the classification. They are ADI, $H_{1}$ likelihood, and pixel mean intensity, with the following three reasons:

1. pixels with single scatterer experience lower ADI, since the amplitudes of pixels subjected to only a single dominant scatterer do not show pronounced fluctuations as a function of baseline; 
2. the $H_{1}$ likelihood tends to be lower if multiple scatterers present;

3. the abovementioned two aspects are based on a reasonable SNR range. Therefore, only relatively bright pixels should be selected as candidates of pixels with double scatterers.

Without the analytical model of the joint probability density function (PDF) of the abovementioned three quantities, we employ the SVM to find a separation of these two classes. Considering our situation of missing classification ground truth, the usage of SVM follows the basically the points below:

- The training data of the SVM classifier is the classification results obtained by SL1MMER (theoretically the closest to ground truth) [12] on a small and representative test area. The SVM classifier takes into account three features: ADI, $H_{1}$ likelihood, and pixel mean intensity.

- Non-linear SVM classifier is applied. Gaussian kernel is used, which is suggested in [13] as a good first try.

- The optimum parameter setting is determined by:

1. Train the classifier using $50 \%$ of the pixels in the small test area.

2. Classify all the pixels in the test area using the trained classifier

3. Repeat this with a geometric sequence of the parameters, and finding the parameters which gives the highest detection rate at a constant false alarm rate.

- The trained SVM classifier is then used to classify all the pixels in the whole scene, based on the three features of each pixel.

\section{Higher order spectral estimation (SVD-Wiener, SL1MMER)}

All pixels classified as double scatterers are processed using SVD-Wiener first, taking into account the prior knowledge of the estimates obtained in the first order spectral estimation. In the SVD-Wiener processing, these pixels are tested through model order selection and are classified to no, single, or double scatterer(s) again. Two layers of elevation and motion parameter estimates are retrieved from the pixels confirmed with double scatterers. The lower layer of elevation estimates is interpolated in range-azimuth dimension to get a ground surface height $s_{0}$ for each pixel (the index of the pixel is 
ignored in $s_{0}$ ).

Since SVD-Wiener has almost no super resolution capability, double scatterers with elevation distance shorter than the Rayleigh resolution $\rho_{s}=\lambda r / 2 \Delta b$ [1] ( $r$ : slant range, and $\Delta b$ : elevation aperture size) will be mis-classified as single scatterer. Therefore, among the detected pixels with single scatterer, those whose elevation estimates with respect to its corresponding ground surface height smaller than $\rho_{s}$ (in practice, we allow some buffer and set it to be $1.5 \rho_{s}$ ) are potential pixels that require super-resolution. In this case, SL1MMER is applied. This decision is marked as the red diamond in the processing flowchart in Figure 1. In the SL1MMER processing and the search range is restricted to $\left[\begin{array}{ll}s_{0} & s_{0}+1.5 \rho_{s}\end{array}\right]$.

\section{VALIDATION WITH REAL DATA}

\section{A. Experiments on number of scatterers discrimination}

This section will evaluate the performance of the scatterer discriminator over a test area in downtown Las Vegas shown in Figure 2. This stack contains 25 TerraSAR-X high resolution spotlight images. Since the missing of ground truth, the SL1MMER result is taken as the reference for comparison.

Firstly, the pixels without scatterer are determined according to Equation (6). The prior probabilities $P_{0}$ and $P_{1,2}$ are set to be both $50 \%$ in the experiment. Then, the remaining pixels are separated using SVM into containing either single scatterer or double scatterers. Figure 3 (a) and (b) are a comparison of the SVM and SL1MMER classification result, with red represents pixels with double scatterers, green for pixels with single scatterer, and blue for no scatterer.

In Figure 3 (a), 47\% of the pixels are discarded as no-scatterers, $36 \%$ are detected as single scatterers, and the rest $17 \%$ are detected as double scatterers. The classification of pixel with no scatterer is almost $100 \%$ correct compared to the SL1MMER result. In the rest of the pixels, the detection rate of pixels with double scatterers is $72.4 \%$, with a false alarm rate of $25.7 \%$, which is why much more pixels are 
detected as double scatterers. However, higher detection rate (consequently higher false alarm rate) is still favored in the processing, because the falsely detected pixels with double scatterers can still be corrected in the higher order spectral estimation, but not for the mis-detected pixels with double scatterers. In order to fully characterize the proposed single and double scatterer(s) detector, its ROC curve is plotted in Figure 4. The blue dots are the experimental results, and the green curve is an analytical fit. Depending on the processing power, the threshold can be shifted towards higher detection rate, affording higher false alarm rate, and therefore more processing effort by more frequently applying the SVD-Winer and the SL1MMER.

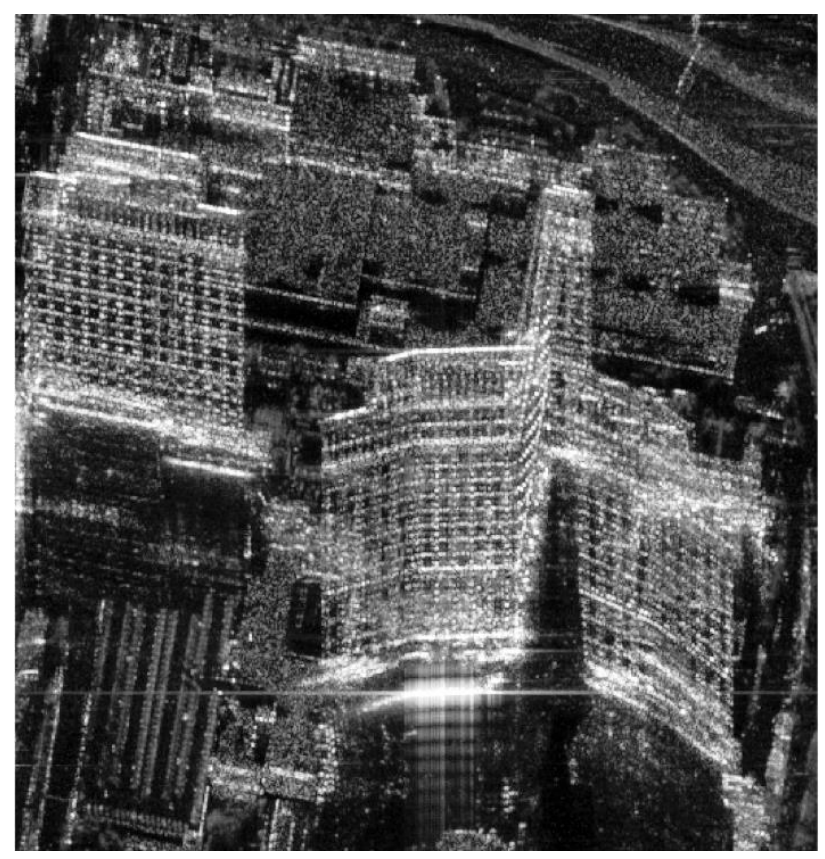

(a)

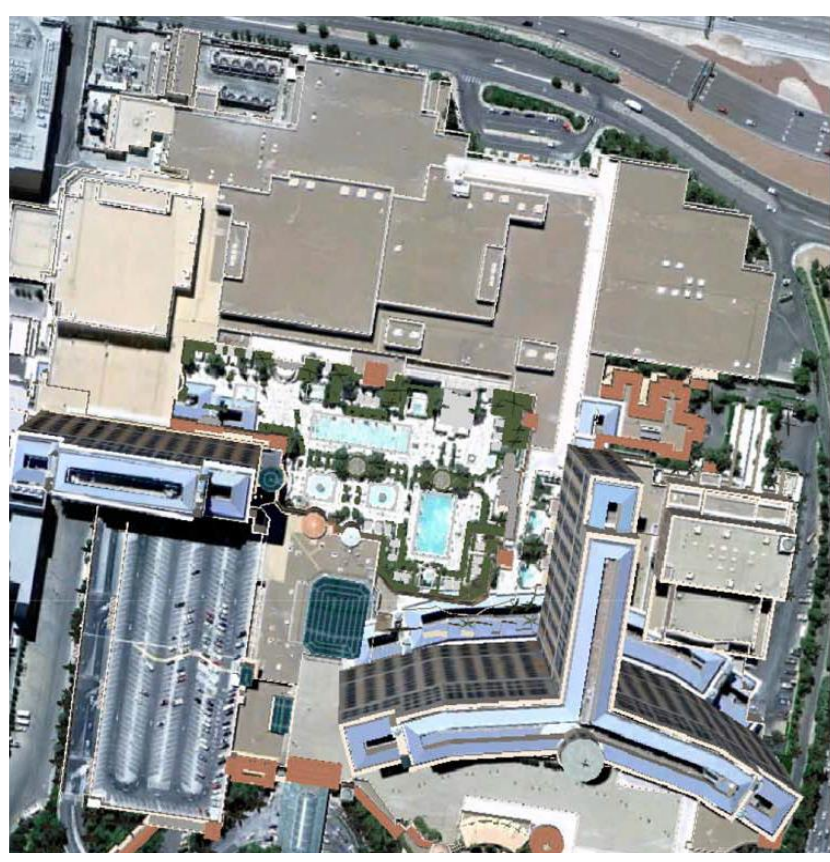

(b)

Figure 2. (a) Amplitude image of the test area in downtown Las Vegas, and (b) optical image from Google Earth of the same area 


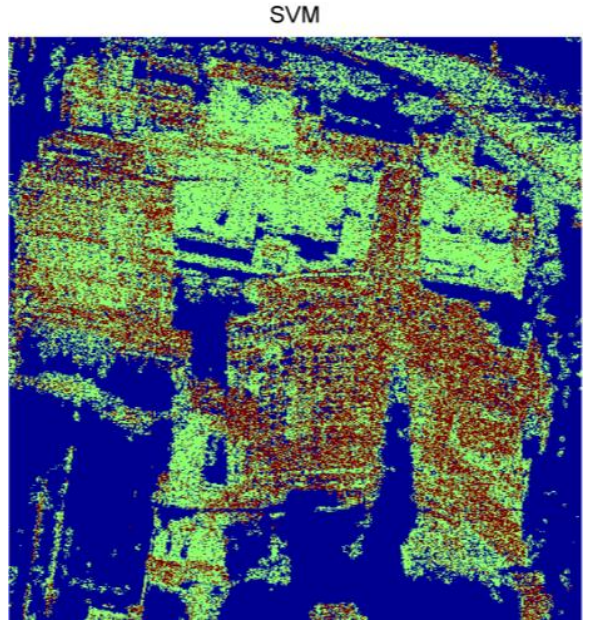

(a)

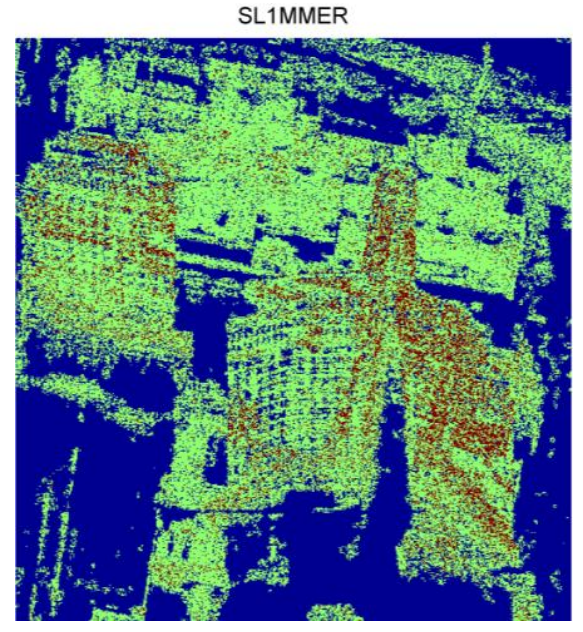

(b)

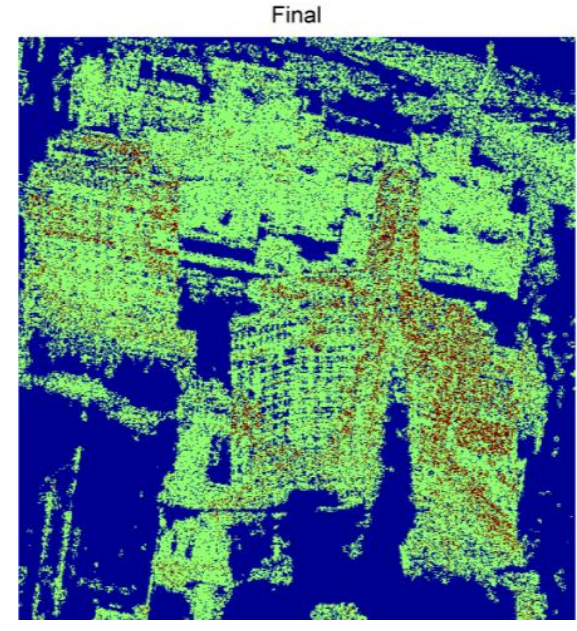

(c)

Figure 3. (a) pre-classification result of the proposed method (likelihood ratio test + SVM), (b) result from the SL1MMER algorithm, and (c) the final classification after the higher order spectral estimation. The red pixels are detected double scatterers, the green are the pixels with single scatterer, and the blue are pixels without coherent scatterer.

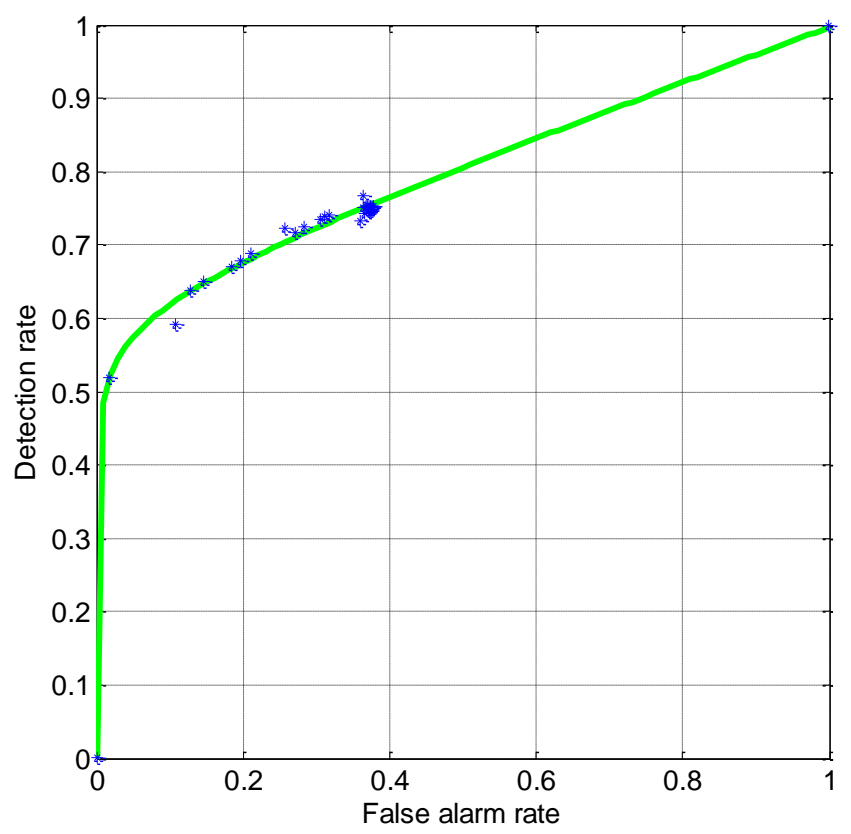

Figure 4. ROC curve of the proposed SVM double scatterers' detector. The blue dots are the best detection rates achieved in the experiments at a specific false alarm rate. The green curve is an analytical fit to the blue dots.

Next, SVD-Wiener is applied on the red pixels shown in Figure 3 (a). Most of them are confirmed as double scatterers, and others are rejected as no- or single scatterer. Among those rejected as single 
scatterers, those having small elevation estimates $\left(60 \mathrm{~m}\right.$ in our case $=1.5$ times of $\left.\rho_{s}\right)$ w.r.t the ground surface height are handed over to SL1MMER for reprocessing. They account for 9\% of the total pixel number in the test area. Then the final corrected classification map of the test area can be obtained, which is shown in Figure 3(c).

For a quantitative measure of the final classification, $98 \%$ of the pixels are corrected classified (the SL1MMER results in Figure 3(b) as reference). The major classification error comes from the mis-detection of pixels with double scatterers. This can be improved by allowing higher detection rates in the double scatterer detection. However, computation effort will also increase correspondingly, since more pixels will be processed by higher order spectral estimation.

\section{B. Analysis on the computational complexity}

This section will discuss the computational complexity of the proposed approach. We define $O(1)$ to be the computation time for one multiplication. The discussion will be focused on the simple case without any motion model. To extend to a single- or multi-component motion model, the computational complexity increases exponentially with the number of dimensions in the system model of the problem.

In the first order spectral estimation, the periodogram consists of multiplication of the measurement vector with the modeled phase, and repeats at different elevation positions. Therefore, the computational complexity is $O(N L)$, where $N$ is the number of images (usually 20 50) and $L$ is the discretization level in the elevation direction (usually 50 200 for TerraSAR-X). In the higher order spectral estimation, the SVD-Wiener needs at least $O\left(N^{2}+N L\right)$ according to the Equation (11) in [4]. The SL1MMER algorithm requires a $L_{1}-L_{2}$ norm optimization which is extremely computationally costly, e.g. the well-known Basic Gradient Projection for Sparse Reconstruction (GPSR-Basic) solver [14] requires at least $O\left(K M L_{s}{ }^{2}\right.$ ), where $K$ is the number of iterations (approximately 20), $M$ is the number of multiplication of a specific matrix in each iteration (modest, usually several), and $L_{s}$ is the elevation discretization level required for sparse reconstruction, which is about 10 times greater than $L$. Our experience is the SL1MMER algorithm is several hundred times slower than SVD-Wiener.

When prior knowledge of the elevation span is available, the $L$ can be restricted to a specific 
elevation range, and hence reduce the computational cost. For instance, assuming a uniform distribution of building heights prior over the processing area, the total processing cost can be reduced to half for SVD-Wiener when $L>>N$, and to one third for SL1MMER. Therefore, it is always more economic to obtain the prior through some low-cost methods before the higher order spectral estimation.

The overall computational cost also depends on the percentage of pixels allocated to each algorithm. As an example, for the test area discussed in Section IIIA, 100\% of the pixels are first processed by a periodogram, $17 \%$ are then passed to SVD-Wiener, and only $9 \%$ are finally processed by SL1MMER. This small percentage together with the restriction of the search space leads to an overall speed-up of about 50 compared to SL1MMER-only processing.

The computational cost of the pixel pre-classification using SVM is negligible compared to the PSI plus TomoSAR processing, and hence ignored in this analysis. Quicker processing can be achieved if no super resolution is required.

\begin{tabular}{|c|c|c|c|c|}
\hline \multirow{2}{*}{ Processing Schemes } & \multicolumn{3}{|c|}{ Percentage of pixels processed by each algorithm } \\
\cline { 2 - 5 } & Periodogram & SVD-Wiener & $\begin{array}{c}\text { SL1MMER } \\
\text { (full elevation } \\
\text { search range) }\end{array}$ & $\begin{array}{c}\text { SL1MMER } \\
\text { (60m elevation } \\
\text { search range) }\end{array}$ \\
\hline $\begin{array}{c}100 \% \text { SL1MMER } \\
\text { (w/o prior) }\end{array}$ & - & - & $100 \%$ & - \\
\hline $\begin{array}{c}100 \% \text { SVD-Wiener } \\
\text { (w/o prior) }\end{array}$ & - & $100 \%$ & - & - \\
\hline Proposed processing chain & $100 \%$ & $17 \%$ (prior) & - & $9 \%$ (w/o prior) \\
\hline
\end{tabular}

Table 1. Overall computational cost of the proposed processing chain, compared to using SL1MMER and SVD-Wiener only.

\section{Large Area Processing: Berlin}

The proposed processing chain is applied on several stacks of TerraSAR-X high resolution spotlight images over Berlin. The following figure is the elevation estimates and a close-up view of one of the stacks which comprises 94 images. In the close-up view of the Berlin central station, the proposed processing chain is able to catch most of the structure. 




(a)

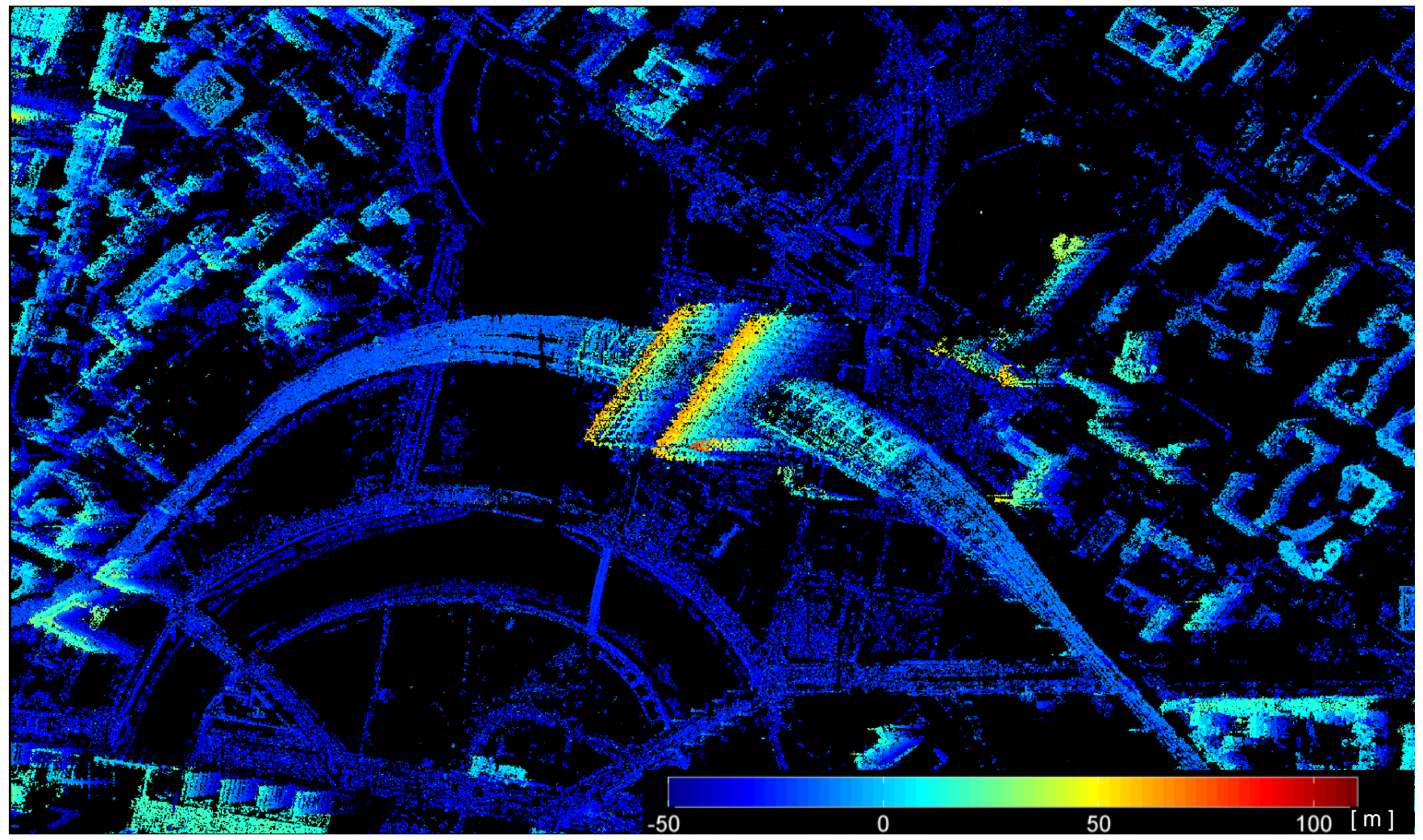

(b)

Figure 5. (a) Elevation estimates of Berlin using the proposed approach, and (b) a close-up over the Berlin central station. 


\section{CONCLUSION \& OUTLOOK}

This article proposed an efficient SAR tomography inversion approach for meter resolution SAR image stacks of urban area. It is the integration of three spectral estimation algorithms: periodogram, SVD-Wiener, and SL1MMER. It has been tested on a number of image stacks of urban areas. Reliable results are obtained, and a speed up factor over fifty is confirmed.

The computational cost is saved in two aspects: 1. prior knowledge of the solution search range is obtained through low cost periodogram, and 2. only pixel with more than one strong scatterer is processed using SVD-Wiener, and SL1MMER is only applied to pixel requires super resolution.

The resulting VHR spaceborne TomoSAR point clouds can be used for multiple applications. For example, individual building monitoring over the whole city, fusion of point cloud from different angles [15], and city 3D model reconstruction from the point clouds [16].

\section{ACKNOWLEDGMENT}

This work is supported by International Graduate School of Science and Engineering, Technische Universität München (Project 6.08: “4D City”), the Helmholtz Association under the framework of the Young Investigators Group "SiPEO” and the German Research Foundation (DFG, Förderkennzeichen

BA2033/3-1). The authors gratefully acknowledge the Gauss Centre for Supercomputing e.V. (www.gauss-centre.eu) for funding this project by providing computing time on the GCS Supercomputer SuperMUC at Leibniz Supercomputing Centre (LRZ, www.lrz.de).

\section{REFERENCES}

[1] A. Reigber, A. Moreira, "First demonstration of airborne SAR tomography using multibaseline L-band data," IEEE Transactions on Geoscience and Remote Sensing, vol. 38, no. 5, 2000, pp. 2142-2152,

[2] F. Lombardini, "Differential tomography: a new framework for SAR interferometry", IEEE Transactions on Geoscience and Remote Sensing, vol. 43, issue. 1, 2005, pp. 37-44. 
[3] G. Fornaro, D. Reale, F. Serafino, "Four-Dimensional SAR Imaging for Height Estimation and Monitoring of Single and Double Scatterers", IEEE Transactions on Geoscience and Remote Sensing, vol. 47, issue. 1, 2009, pp. 224-237.

[4] X. Zhu, R. Bamler, "Very high resolution spaceborne SAR tomography in urban environment", IEEE Transactions on Geoscience and Remote Sensing, vol. 48, issue. 12, 2010, pp. 4296-4308.

[5] A. Ferretti, C. Prati, F. Rocca, "Permanent Scatterers in SAR Interferometry", IEEE Transactions on Geoscience and Remote Sensing, vol. 39, issue. 1, 2001, pp. 8-20.

[6] B. Kampes, Radar Interferometry: Persistent Scatterers Technique. Dordrecht, The Netherlands: Springer, 2006.

[7] X. Zhu, R. Bamler, "Let's do the time warp: multicomponent nonlinear motion estimation in differential SAR tomography”. IEEE Geoscience and Remote Sensing Letters, vol. 8, no. 4, 2011, pp. 735-739.

[8] X. Zhu, R. Bamler, “Tomographic SAR inversion by L1 normregularization-The compressive sensing approach”, IEEE Transactions on Geoscience and Remote Sensing, vol. 48, issue. 10, 2010, pp. 3839-3846.

[9] N. Adam, B. Kampes, M. Eineder, J. Worawattanamateekul, M. Kircher, "The development of a scientific permanent scatterer system", in proceedings of ISPRS Hannover Workshop, Hannover, Germany, 2003, pp. 1-6 (on CD-ROM).

[10] G. Fornaro; A. Pauciullo; F. Serafino, "Deformation monitoring over large areas with multipass differential SAR interferometry: a new approach based on the use of spatial differences", International Journal of Remote Sensing, vol. 30, no.6, 2009, pp 1455-1478.

[11] A. De Maio, G. Fornaro, A. Pauciullo, "Detection of Single Scatterers in Multidimensional SAR Imaging", IEEE Transactions on Geoscience and Remote Sensing, vol.47, no.7, 2009, pp.2284-2297

[12] X. Zhu, R. Bamler, "Demonstration of Super-resolution for Tomographic SAR Imaging in Urban Environment", IEEE Transactions on Geoscience and Remote Sensing, vol. 50, issue. 8, 2012, pp.3150-3157. 
[13] Chih-Wei Hsu, Chih-Chung Chang, and Chih-Jen Lin. A Practical Guide to Support Vector Classification, 2010, http://www.csie.ntu.edu.tw/ cjlin/papers/guide/guide.pdf

[14] M. Figueiredo, R. Nowak, S. Wright, “Gradient Projection for Sparse Reconstruction:Application to Compressed Sensingand Other Inverse Problems", IEEE Journal of Selected Topics in Signal Processing, vol. 1, no. 4, 2007

[15] Y. Wang, X. Zhu, "Feature-based Fusion Of Tomosar Point Clouds From Multi-View Terrasar-X Data Stacks", in proceedings of IGARSS 2013, Melbourne, July 2013

[16] X. Zhu, M. Shahzad, "Facade Reconstruction Using Multiview Spaceborne TomoSAR Point Clouds," IEEE Transactions on Geoscience and Remote Sensing, vol.PP, no.99, pp.1-12, 2013 